\title{
Inter-relação entre COVID-19 e diabetes mellitus: uma revisão sistemática
}

\author{
Interrelationship between COVID-19 and diabetes mellitus: a systematic review \\ Interrelación entre COVID-19 y diabetes mellitus: una revisión sistemática
}

Recebido: 20/01/2021 | Revisado: 25/01/2021 | Aceito: 27/01/2021 | Publicado: 03/02/2021

\author{
Felipe Pereira Marinho \\ ORCID: https://orcid.org/0000-0003-4900-1170 \\ Universidade José do Rosário Vellano, Brasil \\ E-mail: felipe.marinho@aluno.unifenas.br \\ Isabella Santos de Loyola \\ ORCID: https://orcid.org/0000-0002-0066-2018 \\ Universidade José do Rosário Vellano, Brasil \\ E-mail: isabella.loyola@aluno.unifenas.br \\ Igor de Oliveira Freire Monteiro \\ ORCID: https://orcid.org/0000-0002-2480-2927 \\ Universidade José do Rosário Vellano, Brasil \\ E-mail: igor.monteiro@aluno.unifenas.br \\ Tiffani Marioto Castro \\ ORCID: https://orcid.org/0000-0002-3315-9830 \\ Universidade José do Rosário Vellano, Brasil \\ E-mail: tiffani.castro@aluno.unifenas.br \\ Maria das Graças de Souza Carvalho \\ ORCID: https://orcid.org/0000-0001-8726-6327 \\ Universidade José do Rosário Vellano, Brasil \\ E-mail: mariah_roots@hotmail.com \\ José Antônio Dias Garcia \\ ORCID: https://orcid.org/0000-0002-4024-3045 \\ Universidade José do Rosário Vellano, Brasil \\ E-mail: jadiasgarcia@gmail.com \\ Alessandra Cristina Pupin Silvério \\ ORCID: https://orcid.org/0000.0003.2093.2713 \\ Universidade José do Rosário Vellano, Brasil \\ E-mail: alessandrapupin72@gmail.com \\ Gérsika Bitencourt Santos \\ ORCID: https://orcid.org/000-0003-0849-2786 \\ Universidade José do Rosário Vellano, Brasil \\ E-mail: gersika.santos@unifenas.br
}

\begin{abstract}
Resumo
Introdução: Indivíduos com diabetes correm um risco maior de hospitalização e mortalidade resultante de infecções virais, bacterianas e fúngicas. A doença do coronavírus-2019 (COVID-19), causada pela síndrome respiratória aguda grave do coronavírus-2 (SARS-CoV-2), se espalhou rapidamente para os países e custou milhares de vidas até dezembro de 2020. Notavelmente, em vários estudos, o diabetes é uma das comorbidades mais relatadas em pacientes com COVID-19 grave. Objetivo: Discutir sobre a influência do diabetes sobre o prognóstico de pacientes com COVID-19 através de uma revisão literatura. Metodologia: Trata-se de um estudo de revisão sistemática. Para identificar a literatura publicada até dezembro de 2020, foram aplicadas estratégias de buscas individuais nos seguintes bancos de dados eletrônicos: PubMed, Medline, Lilacs e SciELO. Resultados: Pode-se notar que com base nos novos dados clínicos obtidos de pacientes COVID-19, vários mecanismos, como tempestade de citocinas, disfunção pulmonar e endotelial e hipercoagulação, que podem tornar os indivíduos com diabetes mais vulneráveis à COVID-19. Estudos epidemiológicos mostram que o diabetes mal controlado é um fator de risco para várias doenças infecciosas. Conclusão: Dado a importância clínica do diabetes e a natureza pandêmica dos coronavírus, compreender como o diabetes afeta a gravidade da COVID-19 é fundamental para o desenvolvimento de conscientização da população e de tratamentos personalizados de indivíduos afetados pelas doenças.
\end{abstract}

Palavras-chave: COVID-19; Diabetes; Coronavírus; Mecanismos; Infecção; Revisão sistemática.

\section{Abstract}

Introduction: Individuals with diabetes are at a higher risk of hospitalization and mortality from viral, bacterial and fungal infections. Coronavírus-2019 (COVID-19), caused by severe acute respiratory syndrome coronavírus-2 (SARS-CoV-2), spread rapidly to countries and claimed thousands of lives by December 2020. Notably, in several studies, diabetes is one of the most reported comorbidities in patients with severe COVID-19. Objective: Discuss the 
influence of diabetes on the prognosis of patients with COVID-19 through a literature review. Methodology: It is a systematic review study. To identify the literature published until December 2020, individual search strategies were applied to the following electronic databases: PubMed, Medline, Lilacs and SciELO. Results: It can be noted that based on the new clinical data obtained from COVID-19 patients, several mechanisms, such as cytokine storm, pulmonary and endothelial dysfunction and hypercoagulation, which can make individuals with diabetes more vulnerable to COVID-19. Epidemiological studies show that poorly controlled diabetes is a risk factor for several infectious diseases. Conclusion: Given the clinical importance of diabetes and the pandemic nature of coronaviruses, understanding how diabetes affects the severity of COVID-19 is critical to the development of public awareness and personalized treatment for individuals affected by the disease.

Keywords: COVID-19; Diabetes; Coronavirus; Mechanisms; Infection; Systematic review.

\section{Resumen}

Introducción: Las personas con diabetes corren un mayor riesgo de hospitalización y mortalidad por infecciones virales, bacterianas y micóticas. La enfermedad del coronavirus-2019 (COVID-19), causada por el síndrome respiratorio agudo severo coronavirus-2 (SARS-CoV-2), se propagó rápidamente a los países y se cobró miles de vidas hasta deciembre de 2020. Notablemente, en varios estudios, la diabetes es una de las comorbilidades más notificadas en pacientes con COVID-19 grave. Objetivo: Discutir la influencia de la diabetes o el pronóstico de los pacientes con COVID-19 a través de una revisión de la literatura. Metodologia: Es un estudio de revisión sistemática. Para identificar la literatura publicada hasta diciembre de 2020, se aplicaron estrategias de búsqueda individual a las siguientes bases de datos electrónicas: PubMed, Medline, Lilacs y SciELO. Resultados: Se puede observar que en base a los nuevos datos clínicos obtenidos de pacientes con COVID-19, varios mecanismos, como la tormenta de citocinas, disfunción pulmonar y endotelial e hipercoagulación, pueden hacer que los individuos con diabetes sean más vulnerables al COVID-19. Los estudios epidemiológicos muestran que la diabetes mal controlada es un factor de riesgo para varias enfermedades infecciosas. Conclusión: Dada la importancia clínica de la diabetes y la naturaleza pandémica de los coronavirus, comprender cómo la diabetes afecta la gravedad del COVID-19 es fundamental para el desarrollo de la conciencia pública y el tratamiento personalizado para las personas afectadas por la enfermedad.

Palabras clave: COVID-19; Diabetes; Coronavirus; Mecanismos; Infección; Revisión sistemática.

\section{Introdução}

A COVID-19 com desenvolvimento de síndrome respiratória aguda grave, secundária a infecção pelo SARS-CoV2/2019-nCoV, tem levado a um grave problema de saúde pública e econômico (Liu et al., 2020). No final do ano de 2019, a cidade de Wuhan, na província de Hubei, China, apresentou um surto de insuficiência respiratória aguda de pessoas acometidas por um novo coronavírus (Shereen et al., 2020). A partir daí, ocorreu uma rápida disseminação mundial. A Organização Mundial de Saúde (OMS) declarou Emergência de Saúde Pública de Importância Internacional (ESPII) em 30 de janeiro de 2020 e pandemia em 11 de março de 2020 (Bialek et al., 2020).

A diabetes é uma doença inflamatória crônica com múltiplas alterações metabólicas e vasculares que podem afetar a resposta do organismo de diversas formas. A hiperglicemia e a resistência à insulina promovem uma maior síntese de produtos finais de glicação, citocinas pró-inflamatórias e estresse oxidativo, além de estimularem uma produção maior de moléculas de adesão que mediam a inflamação, o que pode implicar em uma maior propensão para infecções (Tamayo et al., 2020).

Evidências epidemiológicas sugerem que diabéticos são considerados grupos de alto risco para doenças infecciosas e que indivíduos com resistência à insulina têm de 50-60\% maior risco de infecção pulmonar (Wang et al., 2020). Diante desta afirmação em alguns estudos e estatísticas foram observados que pacientes diabéticos podem desenvolver a forma grave da COVID-19 (Wang et al., 2020). Estudos mostram que pacientes infectados com COVID-19 e que possuem diabetes são considerados de alto risco e são mais propensos a desenvolverem formas graves e a morrerem (Guo et al., 2020).

Devido a diabetes ser uma doença de alta prevalência no mundo e o vírus SARS-CoV-2 ser uma realidade pandêmica, este artigo tem por objetivo realizar uma revisão sistemática abordando a relação entre COVID-19 e diabetes mellitus para facilitar o entendimento entre estas duas doenças fornecendo dados para melhor condução dos pacientes. Assim, a indagação a ser discutida é quais mecanismos levam a maior gravidade da COVID-19 em pacientes diabéticos. 


\section{Metodologia}

Este estudo trata-se de uma revisão sistemática. As diretrizes do protocolo PRISMA foram seguidas para o relato desta revisão. Foram reunidas as principais evidências que levam os pacientes portadores de diabetes a desenvolverem a forma grave da COVID-19. Os critérios de inclusão estabelecidos foram os estudos relacionados à pacientes com diabetes e com COVID-19. Não houve restrição quanto a data de publicação, idioma ou tipo de estudo.

\subsection{Estratégia de busca}

Para identificar a literatura publicada até dezembro de 2020, foram aplicadas estratégias de buscas individuais nos seguintes bancos de dados eletrônicos: PubMed, Medline, Lilacs, medRxiv, BBO-Dentristry e bioRxiv. As referências dos artigos incluídos foram rastreadas manualmente para artigos com potencial para inclusão no presente estudo.

A expressão utilizada na busca combinou os seguintes termos presentes no Medical Subject Headings (MeSH): 2019 new coronavirus disease, New 2019 Coronavirus Infection, NCoV disease 2019, NCoV infection 2019, Pandid-19 pandemic, COVID-19 Pandemics, COVID-19 virus disease, COVID-19 virus infection, COVID-19, Coronavirus disease 2019, Coronavirus disease-19, SARS Coronavirus 2 infection ou SARS-CoV-2 infection. condições associadas, doença associada, condições coexistentes, doença coexistente, condições concomitantes, doença concomitante, Covid-19 e complicações e diabetes.

\section{Resultados e Discussão}

Foram identificados 1066 estudos dos quais, 4 eram duplicatas. Com base no título e resumo, 1064 estudos foram avaliados, 1032 excluídos e 32 estudos avaliados na etapa do texto completo. Para essa revisão sistemática rápida, 23 estudos foram incluídos. A razão para todas as exclusões foi a não resposta do artigo à pergunta da pesquisa, conforme demonstra o fluxograma segundo o PRISMA (Figura 1). 
Figura 1 - Fluxograma do processo de seleção de dados para a revisão sistemática.

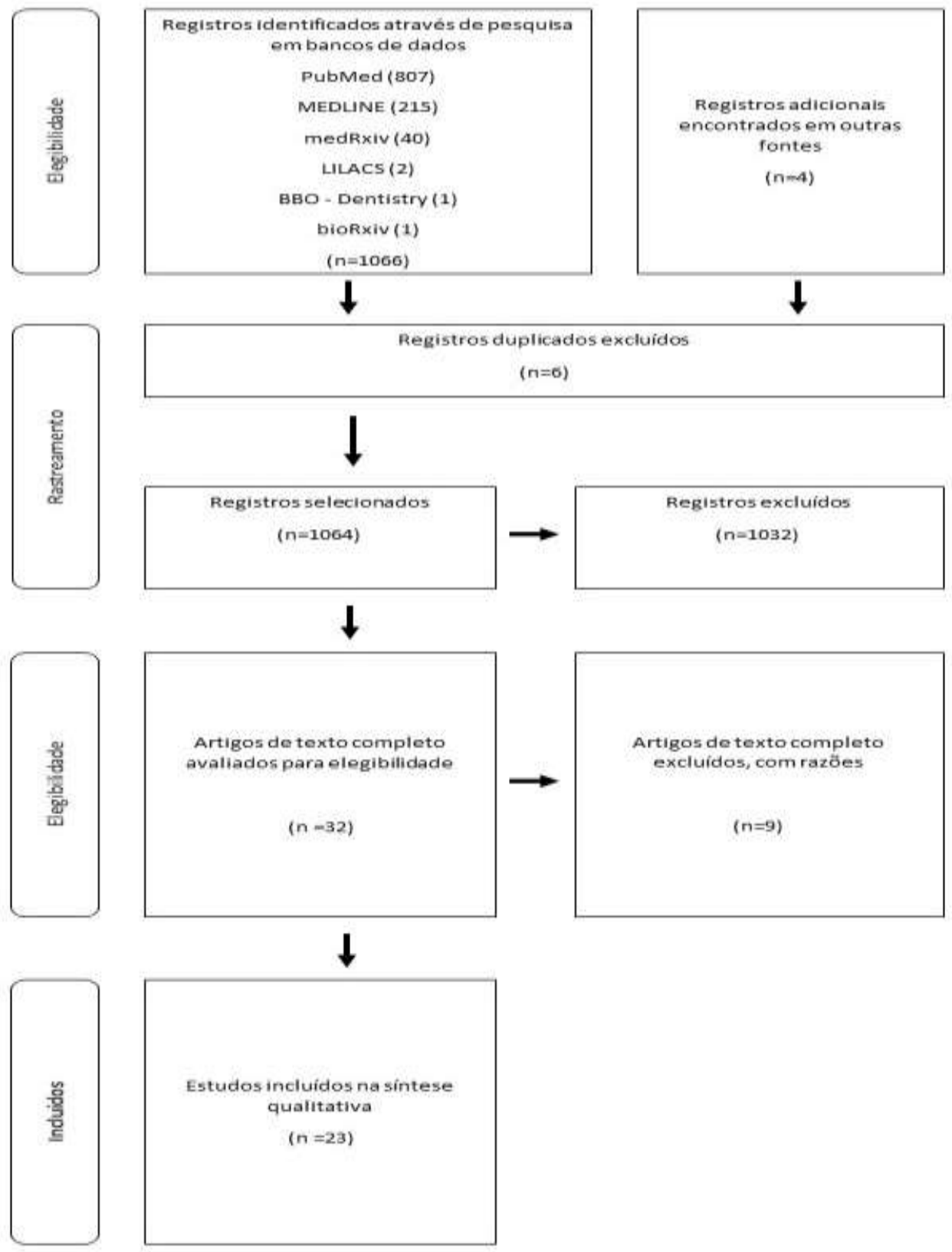

Fonte: Autores.

\section{Epidemiologia em casos de COVID-19 e pacientes diabéticos}

Em um estudo realizado por Guan et al. (2020) que abrangeu 1099 pacientes em 552 hospitais da China continental, investigou-se a presença de fatores de risco em 174 pacientes confirmados com COVID-19. Desses, aqueles com diabetes apresentaram pneumonia severa e um valor mais alto de marcadores de inflamação e coagulação, além de um maior prejuízo cardiovascular (32 vs. 14\%), porém menor febre (59.5 vs. 83.2\%), do que os que não possuíam tal enfermidade. Os dados bioquímicos mostraram nesses pacientes taxas mais elevadas de proteína C reativa (PCR) (32.8 vs. 16.3), velocidade de sedimentação globular (67 vs. 23 ) e D-dímero (1.15 vs. 0.54). Em contraste, o valor absoluto de linfócitos (0.86 vs. 0.97), eritrócitos (3.9 vs. 4.17) e os níveis de hemoglobina (117 vs. 127) foram significantemente mais baixos. Os pacientes com diabetes se caracterizaram por terem mais episódios de vômitos e náuseas. A comparação das tomografias de tórax evidenciou 
a severidade maior das alterações patológicas e, por fim, a mortalidade foi maior entre os pacientes com diabetes (16,3\%) do que os sem diabetes (0\%) (Tamayo, et al., 2020).

Nos primeiros estudos na epidemia do COVID-19 realizados por Huang et al. (2020), foi demostrado que em 41 casos graves de COVID 19, $20 \%$ eram de diabéticos. Em outro estudo feito por Chen et al. (2020) com 99 infectados foi verificado que 52\% apresentavam hiperglicemia. Outro estudo feito por Wang et al. (2020) contou 138 pacientes, $47 \%$ tinha outras comorbidades e $10 \%$ apenas diabetes. Nesse mesmo estudo foi comprovado que nas unidades de tratamento intensivo (UTI) $22 \%$ dos pacientes eram diabéticos. Nas pesquisas coordenadas pelo Yang et al. (2020) no Hospital Jin Yin Tan Wuhan demostraram que na UTI, $17 \%$ dos pacientes eram diabéticos, além de que 35\% com quadro hiperglicêmicos e que a cada 9 pacientes que morrem de COVID-19, 7 eram diabéticos (Wang et al., 2020).

Um estudo maior, realizado por Wu, Tang e Cheng (2020), de 72.314 pacientes com COVID-19 na China os portadores de diabetes tinham uma taxa três vezes maior de mortalidade em comparação com a taxa de mortalidade em pacientes no geral (7,3\% vs 2,3\%). Da mesma forma, de acordo com Tamayo et al. (2020), a mortalidade é cerca de três vezes maior em pessoas com diabetes, em comparação com a mortalidade geral de COVID-19 na China.

Em uma série de casos recentes que incluiu 5.700 pacientes hospitalizados com COVID-19 na área de Nova York, Richardson et al. (2020) relataram que, dos pacientes que morreram, aqueles com diabetes eram mais propensos a receber ventilação mecânica invasiva nos cuidados intensivos em comparação com aqueles sem diabetes.

Estudos epidemiológicos dos três surtos relacionados ao coronavírus (SARS, MERS, COVID-19) identificou diabetes e outras doenças, como hipertensão, doenças cardiovasculares e cerebrovasculares como fatores de risco para infecções graves ou letais. Alqahtani et al. (2020) mostraram que a prevalência de óbito era de 50\% em pacientes com diabetes comparado com a taxa de mortalidade geral de $20 \%$ em 281 casos confirmados de MERS-CoV. Na atual pandemia, a incidência de pacientes em terapia intensiva com diabetes é duas vezes maior em comparação com pacientes não intensivos com COVID-19 (Tamayo et al., 2020).

\section{Mecanismos que correlacionam o prognóstico da COVID-19 em pacientes com diabetes}

Dentre os mecanismos que poderiam contribuir com o aumento da susceptibilidade e mau prognóstico da COVID-19 em pacientes com diabetes, destaca-se: união celular de maior afinidade e entrada de vírus eficiente, eliminação viral diminuída, diminuição da função das células $\mathrm{T}$, aumento da susceptibilidade à hiperinflamação e presença de doença cardiovascular (Tamayo, et al., 2020).

\section{Influência do receptor de enzima conversora de angiotensina 2 (RACE2)}

O receptor de enzima conversora de angiotensina 2 (RACE2), presente na superfície celular de diversos órgãos, é utilizado pelo SARS-Cov-2 para infectar as células humanas. O SARS-CoV-2 adentra as células humanas quando a glicoproteína S “spike”, presente na superfície do vírus, se liga ao RACE2 das células humanas. Após o contágio e a ligação do vírus, a proteína "spike" é clivada por uma protease da célula hospedeira, permitindo o ingresso do vírus na célula e sua replicação. Após a endocitose do complexo viral, a expressão de RACE2 é diminuída (Wiersinga et al., 2020).

No começo da infecção, o vírus visa infectar células nasais, células epiteliais dos brônquios e pneumócitos por meio de sua proteína S que se liga ao RACE2. Para que isso ocorra, a serina protease transmembrana tipo 2 (TMPRSS2) precisa estar presente na célula-alvo para que possa ativar a proteína $\mathrm{S}$ e clivar o RACE2, possibilitando e mediando a entrada do vírus na célula hospedeira. Tanto o RACE2 quanto a TMPRSS2 são expressas nas células-alvo do SARS-CoV-2 para que ele possa infectá-las, sendo significativamente expressas particularmente nas células epiteliais alveolares do tipo 2 (Wu, Tang \& Cheng, 2020). 
O SARS-CoV-2 possui tropismo pelo trato respiratório devido à alta concentração de RACE2, seu receptor de entrada, e pode infectar vários tipos de células epiteliais, incluindo as células epiteliais do tipo 2, responsáveis por evitar o colabamento dos alvéolos. Estudos histopatológicos relataram que o vírus possui tropismo orgânico pelo tecido respiratório, renal, miocárdico, neurológico, faríngeo e gastrointestinal. Além disso, estudo em sequências de RNA viral confirmaram que a expressão de RACE2 é maior nas células do tipo 2 no epitélio alveolar, células secretoras nasais, colangiócitos, colonócitos, queratinócitos do esôfago, epitélio gastrointestinal, células beta-pancreáticas e túbulo contorcido proximal (Gupta et al., 2020; Guo et al., 2020).

Ademais, o diabetes mellitus 2 (DM2) induz a maior expressão de ACE2 no pâncreas e em outros órgãos (pulmão, fígado e coração), o que contribui para uma possível falência de múltiplos órgãos, causada como uma última parte do dano viral direto (Tamayo et al., 2020). Além disso, alguns medicamentos usados por diabéticos, como inibidores da enzima conversora de angiotensina (ECA), agonistas do GLP 1 e estatinas podem aumentar ainda mais os níveis da RACE (Bornstein et al., 2020).

\section{Influência da enzima dipeptidil peptidase-4 (DPP-4)}

Um segundo mecanismo potencial que pode explicar a ligação entre COVID-19 e diabetes envolve a enzima dipeptidil peptidase-4 (DPP-4), que foi identificado como um receptor funcional para o vírus responsável pela MERS (Bornstein et al., 2020). Como a expressão de ECA2 em células alveolares do tipo 2 é comparativamente menor do que em outros tecidos (Hikmet et al., 2020), aumenta as chances de que o SARS-CoV-2 utiliza-se de correceptores, como a DPP-4. Um indício disso é que a DPP-4 está presente no epitélio do trato respiratório inferior, principalmente nas células alveolares tipo 2 (Bassendine et al., 2020).

Tal enzima atua, principalmente, no sistema imune e no metabolismo de glicose e insulina. Em relação à imunidade, ela é capaz de ativar células T e regular a expressão de CD86 e a via NF-kB, além de aumentar a inflamação em casos de DM2 e poder alterar a funcionalidade de citocinas e quimiocinas. Já no âmbito metabólico, ela reduz a secreção de insulina e metabolismo anormal do tecido adiposo visceral, também regulando a glicose pós-prandial. (Iacobellis, 2020).

O modelamento da estrutura da proteína "spike" do SARS-CoV-2 prediz que seu domínio S1 se liga à DPP-4 humana. A DPP-4 é intensamente expressa na glândula salivar, rim, fluído seminal e fígado, também sendo encontrada em altas concentrações nos enterócitos colônicos, capilares, epitélio pulmonar e células imunológicas. Isso indica que o SARS-CoV-2 também pode ser transmitido pela via oral-fecal em humanos. Além disso, fatores que aumentam a expressão de DPP-4 no corpo são: idade avançada, obesidade, baixa taxa de filtração glomerular (TFG), doenças hepáticas e cirrose. Como consequência desses fatores e da atuação da enzima, a DPP-4 é capaz de intensificar o estado inflamatório de pacientes com DM2 e/ou obesidade (Bassendine et al., 2020).

Gliptinas, que são inibidores de DPP-4, podem ser classificados em três classes e são estabelecidos para o tratamento de DM2 ao aumentar a secreção de insulina e diminuir a glicemia (Iacobellis, 2020). Contudo, cada gliptina e suas classes podem possuir efeitos diferentes, sendo que sua atividade anti-inflamatória ao inibir DPP-4 pode ser estudada para, possivelmente, tratar COVID-19 principalmente em pacientes com hiperglicemia (Bassendine et al., 2020).

\section{Alterações metabólicas, hidroeletrolíticas e cardiovasculares do paciente com diabetes}

O índice de massa corporal é um determinante importante do volume pulmonar, mecânica e oxigenação durante a ventilação mecânica, especialmente na posição supina. A maioria dos pacientes com diabetes tipo 2 está vivendo com condições de sobrepeso ou obesidade. Portanto, pacientes com obesidade e diabetes podem estar em risco específico de 
insuficiência ventilatória e complicações durante a ventilação. Além disso, a obesidade é frequentemente associada à inatividade física, levando a resistência à insulina agravada (Bornstein et al., 2020).

A medicação clínica mostrou que a dose de insulina aumentou após o paciente ter sido infectado com SARS-CoV-2, o que mostra que o vírus tem um impacto no metabolismo da glicose do paciente. A desregulação do metabolismo da glicose agrava a diabetes e, em seguida, afeta a gravidade da pneumonia, que funciona como uma amplificação do ciclo (Guo et al., 2020). De acordo com estudos, examinados por Guo et al. (2020), relatou-se que do total de pacientes com diabetes que usavam insulina antes de sua internação por COVID-19, 29,2\% aumentaram as doses de insulina após a saída e 37,5\% dos pacientes que tomavam medicamentos orais antes da entrada ao hospital começaram com a terapia de insulina depois da saída. Isso destaca um controle glicêmico deficiente em pacientes com diabetes durante a internação por SARS-CoV-2 (Tamayo et al., 2020).

Dadas as múltiplas tensões associadas ao COVID-19 incluindo, entre outros, insuficiência respiratória, defeitos na secreção de insulina e a ocorrência frequente de diarreia e sepse, a maioria dos pacientes precisará de insulina. Um cuidado considerável é necessário no balanço hídrico, pois existe o risco de excesso de líquido e isso exacerba o edema pulmonar na região gravemente do pulmão inflamado. Além disso, o equilíbrio de potássio precisa ser cuidadosamente considerado no contexto do tratamento com insulina como hipocalemia, uma característica comum no COVID-19 (possivelmente associado ao hiperaldosteronismo induzido por altas concentrações de angiotensina 2) e pode ser exacerbada após o início da insulina. A elevada hemoglobina A1c em pessoas com diabetes tipo 1 compromete a função imunológica, tornando-os mais suscetíveis a qualquer doença infecciosa. Esses indivíduos precisarão de monitoramento mais intenso e apoio terapêutico para reduzir o risco de descompensação metabólica incluindo CAD, em particular para quem toma sódio, inibidores do co-transportador de glicose 2 (SGLT2). Portanto, a triagem para hiper-inflamação usando marcadores laboratoriais são de importância crucial e também pode ajudar a identificar subgrupos de pacientes para os quais a imunossupressão (esteróides, imunoglobulinas, bloqueio seletivo de citocinas) poderia melhorar o resultado (Bornstein, et al., 2020).

A piroptose induzida por vírus está associada a dano vascular e inflamação, como observado em pacientes com SARS-CoV. Em todas as patologias observadas devido ao COVID-19, o dano não se limitou ao pulmão e ocorreu em vários órgãos, incluindo coração e rins, sugerindo que o SARS-CoV-2 pode infectar células endoteliais vasculares e circular para outros órgãos. De fato, a RACE2 é expressa em vasos sanguíneos e Monteil et al. (2020) recentemente demonstraram que o SARS-CoV-2 poderia infectar diretamente células de vasos sanguíneos em organoides capilares humanos. Como importante, Varga et al. (2020) mostraram a presença de elementos virais e acúmulo de células inflamatórias nas células endoteliais de pacientes com COVID-19, com evidência de morte celular endotelial e inflamatória. Isso sugere que a infecção por SARSCoV-2 pode iniciar a inflamação endotelial em vários órgãos, e a piroptose pode ter um papel importante na lesão celular endotelial e na resposta inflamatória do hospedeiro. Em indivíduos com DT1 ou DT2, a disfunção endotelial é um achado consistente e precede a doença microvascular. O aumento de lesões vasculares, inflamação endotelial e vasoconstrição associadas à disfunção endotelial colocam indivíduos com diabetes em maior risco de endotelite em vários órgãos. A alteração do tônus vascular em direção a uma maior vasoconstrição pode levar a isquemia subsequente dos órgãos, edema tecidual e um estado pró-coagulante. No entanto, é necessário determinar se a insuficiência orgânica observada no COVID-19 é causada por infecção viral direta da vasculatura e de outros órgãos. Além disso, a disfunção endotelial observada em indivíduos com diabetes pode contribuir para a tempestade de citocinas e lesões pulmonares. É relatado que oscilações glicêmicas induzem a produção de citocinas endoteliais e moléculas de adesão que, por sua vez, podem causar extravasamento descontrolado de leucócitos no alvéolo durante a infecção pelo vírus influenza, levando a danos nos pulmões e comprometimento da função respiratória. Se a produção de citocinas a partir de células endoteliais contribui para lesões pulmonares em pacientes com COVID-19 e se isso é agravado em indivíduos com diabetes, permanece por investigar (Erener, 2020). 


\section{Resposta imune e inflamatória do paciente diabético com COVID-19}

A desregulação imunológica, em pacientes diabéticos, induz uma resposta insuficiente ao Interferon (IFN) tipo I, secreção aberrante de citocinas pró-inflamatórias por macrófagos alveolares, e uma subsequente disfunção das células T CD4 + e células T CD8 +. A secreção do IFN é a resposta crítica para suprimir a replicação viral, disseminação em um estágio inicial e indução de resposta imune adaptativa eficaz. Nos casos graves ou letais de infecção por SARS-CoV ou MERS-CoV, o aumento do influxo de neutrófilos e monócitos-macrófagos é observado e a resposta à infecção viral pelo IFN tipo I é suprimida (Erener; 2020).

A inflamação metabólica, que ocorre no diabetes mellitus, hiperglicemia e obesidade irá reduzir a habilidade do corpo de rastrear a infecção e combatê-la, dificultando o processo de cura e prolongando a recuperação, além de induzir a replicação do SARS-CoV-2 (Bornstein et al., 2020). A desregulação da resposta imune e síndrome de liberação de citocinas, devido a uma super-reação do sistema imunológico nas células T caracterizam uma das apresentações da gravidade do COVID (Guo et al; 2020).

Dados de pacientes infectados com SARS-CoV-2 também indicam um aumento no total de neutrófilos, uma redução no total de linfócitos e um aumento no IL-6 sérico e na proteína c-reativa (PCR) (Guo et al., 2020). Elevados níveis de marcadores inflamatórios como proteína C reativa, ferritina, D-dímero, fibrogênio são indicativos de quadro grave ou morte em pacientes com COVID-19 (Gupta et al., 2020).

Aumento de neutrófilos e diminuição de linfócitos também se correlacionam com a gravidade da doença e a morte, já que os linfócitos são responsáveis pela imunidade celular. Caso o vírus infecte linfócitos T, ele poderá causar linfopenia. Além disso, a resposta inflamatória ao vírus, tanto pelo sistema imunológico inato quanto ao adaptativo, causará o comprometimento da linfopoiese e estimula a apoptose de linfócitos. Por fim, os pacientes que necessitam de terapia intensiva apresentam níveis plasmáticos mais elevados de citocinas pró-inflamatórias (IP-10, MCP-1, MIP-1A e TNF $\alpha$ ) (Wang et al., 2020; Chiappetta et al., 2020).

O excesso na produção de citocinas tipo 2 pode causar uma deficiência no controle de replicação viral, causando um estado ainda mais prolongado de resposta pró-inflamatória, potencialmente resultando em pior diagnóstico. Ademais, pacientes obesos possuem uma expressão maior de ácido ribonucleico para citocinas pró-inflamatórias, assim como fator de necrose tumoral-alfa e interleucinas, elementos cruciais na síndrome metabólica. A resposta de defesa inata em pacientes obesos é alterada e conduz para uma primeira linha defensiva irregular, resposta inflamatória aumentada e células de resposta $\mathrm{T}$ anormais. Além disso, o papel da interleucinas-6 é fundamental para mediar a resposta de fase aguda e isso é um valioso biomarcador de sepse e injúria em vários órgãos (Chiappetta et al., 2020).

Os macrófagos do tecido adiposo geralmente expressam mais marcadores M2. Kratz et. al., (2014) mostraram que marcadores clássicos de ativação de macrófagos estão ausentes nos macrófagos do tecido adiposo de seres humanos obesos e a disfunção metabólica leva à um fenótipo pró-inflamatório distinto nos macrófagos do tecido adiposo podendo assim contribuir para a gravidade da doença em questão. As células "Natural Killer" (NK), são linfócitos efetores do sistema imunológico inato, que rapidamente excluem células tumorais infectadas por vírus sem sensibilização prévia. Embora existam algumas discrepâncias entre os estudos, algumas evidências sugerem que a atividade das células NK é reduzida em indivíduos com DM2. Delemaire et al., (1997) relataram uma diminuição e alteração das células NK em pacientes obesos com níveis elevados de glicemia de jejum. Essa alteração pode contribuir para a maior gravidade nos pacientes diabéticos e obesos com a COVID19 (Erener, 2020).

Alguns CoV, como o SARS-CoV, infectam diretamente macrófagos e células T. Para iniciar a resposta antiviral, as células imunitárias inatas necessitam reconhecer a invasão do vírus, frequentemente através de padrões moleculares associados a patógenos (PAMP). A resposta do IFN tipo 1 à infecção viral por SARS-CoV y MERS-CoV está suprimida. Não se sabe se 
essa supressão também se faz presente com o SARS-CoV-2, embora possa ser possível, mas especulativo, devido à semelhança na sequência genômica do SARS-CoV-2 com o SARS-CoV e o MERS-CoV (79 e 50\%, respectivamente). Dessa forma, percebe-se que uma resposta imunitária tardia ou suprimida se associa com a gravidade do dano pulmonar (Tamayo et al., 2020).

Indivíduos com diabetes apresentam baixa inflamação crônica, o que pode levar a um recrutamento exagerado de macrófagos, monócitos e células T, promovendo mais inflamação em um ciclo de feedback. A superprodução de citocinas próinflamatórias pode eventualmente danificar a infraestrutura pulmonar. A tempestade de citocinas resultante pode iniciar múltiplas coagulações sistêmicas. No diabetes, o endotélio mostra marcadores de inflamação com aumento de células imunes, citocinas, potencialmente exacerbando a tempestade de citocinas e lesões pulmonares. O SARS-CoV2 pode infectar diretamente células endoteliais através dos receptores ACE2 presentes nas células endoteliais. A alteração do tônus vascular em direção a mais vasoconstrição em pacientes com diabetes pode agravar a subsequente isquemia de órgãos, edema tecidual e um estado pró-coagulante durante a infecção por COVID-19 (Erener, 2020; Tamayo et al., 2020).

\section{Complicações da COVID-19 em pacientes diabéticos}

Diante da expressão pincipalmente dos RACE2 ocorrer nos pulmões e pâncreas, podemos ter consequências nesses órgãos. No caso do pâncreas, a entrada de SARS-CoV-2 pode danificar as ilhotas pancreáticas e as células beta pancreáticas, reduzindo a secreção de insulina, causando hiperglicemia e possível diabetes. Além disso, a diminuição da quantidade de RACE2 pode causar excesso de angiotensina 2, podendo impedir a secreção de insulina (Chee \& Yeoh, 2020), como mencionado anteriormente.

Quando a atividade da enzima conversora de angiotensina 1 (ECA1) diminui e a da ECA2 aumenta, esse excesso de angiotensina 2 também estimula a secreção de aldosterona ao interagir com receptores de angiotensina 1 (RACE1) e RACE2. Isso irá causar aumento da pressão sanguínea e aumento da permeabilidade vascular local, assim como aumentando o risco de hipocalemia (Bornstein et al., 2020), fazendo com que possa ser necessário a ingestão de uma suplementação de potássio pelo paciente para continuar o tratamento da supressão de corpos cetônicos pela infusão de insulina intravenosa (Chee \& Yeoh, 2020; Cheng et al., 2020).

A presença de RACE2 em grande parte dos órgãos do corpo permite visualizar o motivo da facilidade de espalhamento do vírus de forma sistêmica, além de justificar em parte a falência múltipla de órgãos causada pelo COVID-19 (Hikmet et al., 2020)

Pacientes diabéticos infectados com SARS-CoV-2, além do comprometimento da imunidade pelo estado inflamatório crônico já discutido, estimulam hormônios que promovem a produção de glicose hepática, liberação reduzida de insulina, cetoacidose e resistência à insulina. Manifestações extrapulmonares da COVID-19 podem estar relacionadas com complicações diabéticas, como redução da função renal, estado pró-trombótico e de coagulação elevados, disfunção cardíaca e injúria nos hepatócitos. Já a obesidade reduz o volume dos pulmões e aumenta a resistência da passagem do ar. Além disso, o tecido adiposo funciona como um órgão endócrino e, em um paciente obeso, causa uma grande liberação de citocinas, fatores de necrose tumoral e interleucinas, o que corrobora para uma resposta inflamatória frente a doença (Gupta et al., 2020).

\section{Coagulação e trombose no paciente diabético com COVID-19}

Os níveis anormais de D-Dímero e de fibrogênio no sangue durante os primeiros estágios de infecção são reflexos da tempestade inflamatória. A inflamação juntamente com hipóxia causada pele vírus no pulmão e a presença de D-Dímero ativam a trombina diretamente, o que irá iniciar a via de coagulação externa, causando um estado hipercoagulação que 
contribui para as altas taxas de complicações trombóticas no COVID-19. A alta expressão de RACE2 na superfície endotelial perpetua um ciclo vicioso de endotelite, o que promove um trombo inflamação (Gupta, et al., 2020; Guo et al., 2020).

A forma grave da COVID-19 também está associada a um aumento significativo do risco de desenvolver trombose venosa profunda, embolia pulmonar e lesão miocárdica (Wu, Tang \& Cheng, 2020). Visto isso, indivíduos diabéticos têm um risco aumentado para uma resposta inflamatória mais grave, eles podem estar em maior risco de sofrer alterações de coagulação. Notavelmente, indivíduos com diabetes tipo 1 ou tipo 2 apresentam um aumento significativo dos marcadores de hipercoagulação e fibrinólise no plasma. Além disso, a hiperglicemia demonstrou aumentar a coagulação, segundo um estudo de Stegenga et al. (2008), no qual os autores concluíram que a hiperglicemia leva a um estado mais eminente de coagulação. Portanto, pacientes diabéticos são mais vulneráveis a eventos trombóticos durantes ataques inflamatórios (como é o caso da infecção do SARS-CoV-2) (Erener, 2020).

\section{COVID-19 e o desenvolvimento de hiperglicemias}

O SARS-CoV-2 induz a produção de citocinas e quimocinas, podendo levar a uma tempestade inflamatória e à danificação das células pancreáticas, podendo desencadear diabetes e agravar DM2 (Mallapaty, 2020). Durante a infecção, o vírus pode destruir as ilhotas através dos RACE2, agravando a diabetes (Guo et al., 2020).

O SARS-Cov-2 pode desencadear e agravar DM, devido a destruição das células beta e alfa pancreáticas, responsáveis pela produção de insulina e glucagon, respectivamente, após infectá-las. A hiperglicemia causada pelo vírus pode ser um estado de diabetes transitório pela disfunção aguda causada nas células beta, conduzindo a estados de hiperglicemia e DM2 transitável, que podem se tornar crônicos (Mallapaty, 2020).

A infecção por SARS-CoV-2 desencadeia situações de maior estresse em pessoas com diabetes, o que gera um aumento na liberação de hormônios hiperglicêmicos, como glicocorticoides e catecolaminas, favorecendo um aumento da glicose sanguínea. Em um estudo realizado na China, em 2003, foi identificado o desenvolvimento de diabetes em pacientes que não a possuíam antes do contágio por SARS, com observação de comprometimento das ilhotas pancreáticas. De forma anedótica, Joshua Millers reporta casos de pacientes diabéticos e infectados por SARS-CoV-2 com agravamento de deficiência insulínica e aumento de infusões de insulina. Já Jean-François Gautier relata caso de destruição total das células beta pancreáticas. A glicotoxicidade pode ser manifestada como disglicemia até cetoacidose diabética (Tamayo, et al., 2020).

Embora o RACE2 compartilhe algumas características com o RACE1, ele não é inibido por inibidores da ECA (IECA). IECA e bloqueadores do receptor de angiotensina (BRAs) regulam positivamente a expressão de RACE2. Portanto, foi postulado que o uso de IECA/ BRA pode facilitar a entrada de SARS-CoV-2, facilitando a entrada viral nas células hospedeiras (Ma \& Holt, 2020), causando lesões e deficiência de insulina, contribuindo para a hiperglicemia. Além disso, o polimorfismo do gene RACE2 foi associado ao aumento do risco de diabetes e de doença cardiovascular e isso também pode predizer a susceptibilidade à infecção grave por $\mathrm{CoV}(\mathrm{Wu}$, Tang \& Cheng, 2020). Em contraste, a regulação positiva de RACE2 pode aumentar os níveis de angiotensina, montando um efeito anti-inflamatório (Wu, Tang \& Cheng, 2020). No entanto, baixos níveis de angiotensina II (devido à inibição de IECA) pode atenuar esse benefício. Assim, ainda não está claro qual o equilíbrio entre benefício e risco e se isso pode continuar ou interromper a inibição da ECA.

Após um período de incubação de 2 a 14 dias (média de 5 dias), a maioria das pessoas com COVID-19 apresentará lesão de órgão como pulmão, rim, coração, células das ilhotas pancreáticas e deficiência de insulina (Wu, Tang \& Cheng, 2020).

O Quadro 1 apresenta de forma resumida os artigos incluidos nesta revisão. 
Quadro 1 - Apresentação dos artigos incluídos na pesquisa.

\begin{tabular}{|c|c|c|c|c|c|}
\hline Referência & Tipo de estudo & $\begin{array}{l}\text { Sujeitos/ } \\
\mathbf{n}\end{array}$ & Local & Resultado/ conclusão & Observações \\
\hline $\begin{array}{l}\text { Ayres, J.S. A metabolic handbook for } \\
\text { the COVID-19 pandemic. Nat } \\
\text { Metab 2, 572-585 (2020) }\end{array}$ & $\begin{array}{l}\text { Diretriz } \\
\text { (GuideLine or } \\
\text { HandBook) }\end{array}$ & N/A & $\begin{array}{l}\text { Não se } \\
\text { aplica } \\
\text { (N/A) }\end{array}$ & $\begin{array}{l}\text { O importante em uma } \\
\text { pandemia é descobrir como } \\
\text { ocorre, quais as consequências } \\
\text { e como tratar. Esse artigo } \\
\text { aborda todas essas questões. }\end{array}$ & $\begin{array}{l}\text { Explica a fisiopatologia da } \\
\text { infecção, o prognóstico e os } \\
\text { tratamentos. }\end{array}$ \\
\hline $\begin{array}{l}\text { Wang W, Lu J, Gu W, Zhang Y, Liu J, } \\
\text { Ning G (2020). Care for diabetes with } \\
\text { COVID-19: advice from China. Journal } \\
\text { of Diabetes. 12(5), 417-419. }\end{array}$ & Comentário & 278 & China & $\begin{array}{l}\text { Diabetes e hiperglicemia } \\
\text { pioram o prognóstico do covid- } \\
19\end{array}$ & $\begin{array}{l}\text { Diabéticos possuem } 50 \% \\
\text { mais chance de virem a óbito } \\
\text { decorrentes do SARS-CoV } 2 .\end{array}$ \\
\hline $\begin{array}{l}\text { Chiappetta, S., Sharma, A.M., Bottino, } \\
\text { V. et al. (2020) COVID-19 and the role } \\
\text { of chronic inflammation in patients with } \\
\text { obesity. Int J Obes. 44, 1790-1792. }\end{array}$ & $\begin{array}{l}\text { Comunicação } \\
\text { breve }\end{array}$ & 33 & N/A & $\begin{array}{l}\text { Paciente obesos possuem um } \\
\text { processor inflamatório mais } \\
\text { grave e mais duradouro. }\end{array}$ & $\begin{array}{l}\text { A resposta de defesa inata em } \\
\text { pacientes obesos é alterada e } \\
\text { conduz para uma primeira } \\
\text { linha defensiva alterada, } \\
\text { resposta inflamatória } \\
\text { aumentada e células de } \\
\text { resposta } T \text { anormais. }\end{array}$ \\
\hline $\begin{array}{l}\text { Guo W, Li M, Dong Y, Zhou H, Zhang } \\
\text { Z, Tian C, Qin R, Wang H, Shen Y, Du } \\
\text { K, Zhao L, Fan H, Luo S, Hu D (2020). } \\
\text { Diabetes is a risk factor for the } \\
\text { progression and prognosis of COVID- } \\
\text { 19. Diabetes Metab Res Rev; e3319. }\end{array}$ & Artigo de revisão & 174 & China & $\begin{array}{l}\text { Diabetes deve se considerado } \\
\text { um fator de alto risco para um } \\
\text { mal prognóstico de covid- } 19 \text {. } \\
\text { Isso é comprovado por } 24 \\
\text { pacientes diabéticos que foram } \\
\text { acompanhados nesse estudo. }\end{array}$ & \\
\hline $\begin{array}{l}\text { Bornstein, S.R. et al. (2020). Endocrine } \\
\text { and metabolic link to coronavirus } \\
\text { infection. Nat Rev Endocrinol 16, 297- } \\
298 \text {. }\end{array}$ & Comentário & N/A & N/A & $\begin{array}{l}\text { Essa pesquisa mostra que o } \\
\text { SARS-Cov-2 pode desencadear } \\
\text { diabetes mellitus tipo } 1 \text {, devido } \\
\text { a destruição de algumas células } \\
\text { que controlam a glicemia. }\end{array}$ & \\
\hline $\begin{array}{l}\text { Gupta, A., Madhavan, M.V., Sehgal, K. } \\
\text { et al. (2020). Extrapulmonary } \\
\text { manifestations of COVID-19. Nature } \\
\text { Medicine } 26,1017-1032 .\end{array}$ & Artigo de revisão & N/A & N/A & $\begin{array}{l}\text { Explica a fisiopatologia do } \\
\text { covid-19 em outros tecidos } \\
\text { (neurológico, renal, hepático, } \\
\text { gastrointestinal, vascular, } \\
\text { cardíaco, endócrino e } \\
\text { dermatológico) fora do pulmão } \\
\text { e o prognóstico. }\end{array}$ & \\
\hline $\begin{array}{l}\text { Cheng H, Wang Y, Wang G-Q. } \\
\text { Organ-protective effect of } \\
\text { angiotensin-converting enzyme } 2 \text { and } \\
\text { its effect on the prognosis of } \\
\text { COVID-19. J Med Virol. 2020;92:726- } \\
730\end{array}$ & Artigo de revisão & N/A & N/A & $\begin{array}{l}\text { A vacina à base de proteína de } \\
\text { pico ACE2 e rhACE2 podem se } \\
\text { tornar uma das abordagens } \\
\text { mais promissoras para o } \\
\text { tratamento futuro e melhorar o } \\
\text { prognóstico de pacientes com } \\
\text { COVID - } 19 \text {. }\end{array}$ & $\begin{array}{l}\text { Explica toda a relação das } \\
\text { enzimas conversoras de } \\
\text { angiotensina (ACE e ACE2) } \\
\text { com relação a fisiopatologia } \\
\text { do COVID-19. }\end{array}$ \\
\hline $\begin{array}{l}\text { Wiersinga, W. J. et al. (2020). } \\
\text { Pathophysiology, } \\
\text { Transmission, } \\
\text { Diagnosis, and Treatment of } \\
\text { Coronavirus Disease } 2019 \text { (COVID- } \\
\text { 19): A Review. JAMA, 324(8), 782- } \\
\text { 793. }\end{array}$ & Artigo de revisão & N/A & N/A & & $\begin{array}{l}\text { Explica toda a fisiopatologia } \\
\text { do covid, sua forma de } \\
\text { transmissão, diagnóstico e } \\
\text { tratamento. }\end{array}$ \\
\hline $\begin{array}{l}\text { Margarita Torres-Tamayo, et al.: } \\
\text { Infección por coronavirus en pacientes } \\
\text { con diabetes. Arch Cardiol Mex. } \\
\text { 2020;90(Supl). }\end{array}$ & Artigo de revisão & N/A & $\begin{array}{l}\text { Cidade } \\
\text { do } \\
\text { México }\end{array}$ & $\begin{array}{l}\text { Mostra a epidemiologia da } \\
\text { prevalência e mortalidade de } \\
\text { COVID } 19 \text { na população em } \\
\text { geral e na população com DM, } \\
\text { associando a presença de DM } \\
\text { com uma maior mortalidade e } \\
\text { maior necessidade de cuidados } \\
\text { intensivos durante o contágio. }\end{array}$ & $\begin{array}{l}\text { Explica a fisiopatologia } \\
\text { relacionada à ligação do } \\
\text { SARS-CoV-2 a receptores } \\
\text { em indivíduos com DM, bem } \\
\text { como a resposta imune no } \\
\text { organismo. Também aborda } \\
\text { o tratamento ambulatorial e } \\
\text { hospitalar recomendados } \\
\text { para esses tipos de pacientes. }\end{array}$ \\
\hline
\end{tabular}




\begin{tabular}{|c|c|c|c|c|c|}
\hline $\begin{array}{l}\text { BORNSTEIN et al (2020). Practical } \\
\text { recommendations for the management } \\
\text { of diabetes in patients with COVID-19. } \\
\text { The Lancet Diabetes \& } \\
\text { Endocrinology. doi:10.1016/s2213- } \\
8587(20) 30152-2\end{array}$ & Personal View & N/A & $\begin{array}{l}\text { Publicad } \\
\text { o online } \\
\text { na The } \\
\text { Lanced } \\
\text { Diabetes } \\
\& \\
\text { Endocrin } \\
\text { ology }\end{array}$ & $\begin{array}{l}\text { Pacientes com diabetes têm um } \\
\text { aumento do risco de } \\
\text { complicações graves, incluindo } \\
\text { síndrome da dificuldade } \\
\text { respiratória do adulto e falência } \\
\text { de múltiplos órgãos. }\end{array}$ & $\begin{array}{l}\text { Especialistas na área de } \\
\text { diabetes e endocrinologia } \\
\text { fornecem algumas } \\
\text { orientações e recomendações } \\
\text { práticas para a gestão da } \\
\text { diabetes durante a pandemia. }\end{array}$ \\
\hline $\begin{array}{l}\text { Erener S, Diabetes, Infection Risk And } \\
\text { Covid-19, Molecular Metabolism, } \\
\text { https://doi.org/10.1016/j.molmet.2020.1 } \\
01044 \text {. }\end{array}$ & Artigo de revisão & N/A & N/A & $\begin{array}{l}\text { Pessoas com diabetes e } \\
\text { comorbidades relacionadas } \\
\text { apresentam maior risco de } \\
\text { complicações relacionadas ao } \\
\text { COVID-19. }\end{array}$ & \\
\hline $\begin{array}{l}\text { Wu, Z., Tang, Y., \& Cheng, Q. (2020). } \\
\text { Diabetes increases the mortality of } \\
\text { patients with COVID-19: a meta- } \\
\text { analysis. Acta Diabetologica. } \\
\text { doi:10.1007/s00592-020-01546-0 }\end{array}$ & Artigo de revisão & N/A & China & $\begin{array}{l}\text { Os indivíduos com DM2 } \\
\text { exigiam mais intervenções } \\
\text { médicas e teve uma } \\
\text { mortalidade significativamente } \\
\text { maior e lesão de múltiplos } \\
\text { órgãos do que os indivíduos } \\
\text { não diabéticos. }\end{array}$ & \\
\hline $\begin{array}{l}\text { Chee, Y. J., Ng, S., \& Yeoh, E. ( } 2020) \text {. } \\
\text { Diabetic ketoacidosis precipitated by } \\
\text { Covid- } 19 \text { in a patient with newly } \\
\text { diagnosed diabetes mellitus. Diabetes } \\
\text { research and clinical practice, } 164, \\
108166\end{array}$ & Comentário & N/A & $\begin{array}{l}\text { Singapur } \\
\text { a }\end{array}$ & $\begin{array}{l}\text { A ECA2 é muito expressa no } \\
\text { pâncreas e pulmões. Isso faz } \\
\text { com que o vírus possa entrar } \\
\text { nesses locais e, } \\
\text { consequentemente, diminuir a } \\
\text { secreção de insulina e danificar } \\
\text { o parênquima pulmonar, } \\
\text { causando, também, cetoacidose } \\
\text { diabética. }\end{array}$ & $\begin{array}{l}\text { Artigo curto que mostra } \\
\text { resumidamente os } 2 \\
\text { mecanismos que o vírus pode } \\
\text { utilizar para diminuir a } \\
\text { secreção de insulina. }\end{array}$ \\
\hline $\begin{array}{l}\text { Bornstein, S.R. et al. (2020). Endocrine } \\
\text { and metabolic link to coronavirus } \\
\text { infection. Nat Rev Endocrinol 16, 297- } \\
298 \text {. }\end{array}$ & Comentário & N/A & N/A & $\begin{array}{l}\text { Pacientes com DM2 e } \\
\text { Síndrome Metabólica têm suas } \\
\text { chances de mortalidade pelo } \\
\text { COVID-19 aumentas. A DM2 e } \\
\text { hiperglicemia causam constante } \\
\text { estado de inflamação, levando a } \\
\text { comprometimento de funções } \\
\text { do corpo e a quadros que } \\
\text { pioram casos de COVID-19. }\end{array}$ & $\begin{array}{l}\text { O artigo fala sobre os } \\
\text { mecanismos que DM2 e } \\
\text { hiperglicemia causam: } \\
\text { tempestade de citocinas } \\
\text { inflamatórias, desregulação } \\
\text { da resposta imune, na falha } \\
\text { de múltiplos órgãos e } \\
\text { patologia pulmonar mais } \\
\text { agressiva e prolongada. Ele } \\
\text { também comenta sobre } \\
\text { algumas formas de combate } \\
\text { ao vírus e sobre a serina } \\
\text { protease TMPRSS2, que é } \\
\text { necessária para o vírus } \\
\text { infectar a célula. }\end{array}$ \\
\hline $\begin{array}{l}\text { Iacobellis, Gianluca. (2020). COVID-19 } \\
\text { and diabetes: Can DPP4 inhibition play } \\
\text { a role?. Diabetes Research and Clinical } \\
\text { Practice. } 163,108125\end{array}$ & Comentário & N/A & $\begin{array}{l}\text { Estados } \\
\text { Unidos }\end{array}$ & $\begin{array}{l}\text { Artigo descreve sucintamente } \\
\text { sobre o mecanismo de atuação } \\
\text { da DPP-4 no corpo humano e } \\
\text { como ela interage com o } \\
\text { COVID-19, citando } \\
\text { brevemente sua atuação com o } \\
\text { MERS-CoV. }\end{array}$ & \\
\hline $\begin{array}{l}\text { Hikmet, F. et al. (2020). The protein } \\
\text { expression profile of ACE } 2 \text { in human } \\
\text { tissues. Mol Syst Biol, } 16(7), 1-16 \text {. }\end{array}$ & Artigo de revisão & N/A & Suécia & $\begin{array}{l}\text { Artigo identifica o padrão da } \\
\text { expressão de ECA2 e seus } \\
\text { receptores em mais de } 150 \\
\text { tipos celulares diferentes, } \\
\text { abrangendo todos os principais } \\
\text { órgãos e tecidos do corpo } \\
\text { humano. }\end{array}$ & $\begin{array}{l}\text { Artigo usa da imuno- } \\
\text { histoquímica e do perfil de } \\
\text { transcrição da ECA2 em seus } \\
\text { estudos. }\end{array}$ \\
\hline $\begin{array}{l}\text { Bassendine, M. F. et al. (2020). } \\
\text { COVID-19 and comorbidities: A role } \\
\text { for dipeptidyl peptidase } 4 \text { (DPP4) in } \\
\text { disease severity?. Journal of Diabetes. } \\
\text { 12(9), 649-658. }\end{array}$ & Artigo de revisão & N/A & $\begin{array}{l}\text { Reino } \\
\text { Unido }\end{array}$ & $\begin{array}{l}\text { Artigo dialoga brevemente } \\
\text { sobre o papel da enzima DPP-4 } \\
\text { no MERS-CoV e descreve } \\
\text { extensamente sobre a relação } \\
\text { entre DPP-4 e COVID-19. }\end{array}$ & $\begin{array}{l}\text { O artigo descreve sobre o } \\
\text { papel da DPP-4 em casos de } \\
\text { COVID-19 e possíveis } \\
\text { comorbidades ligadas a essa } \\
\text { enzima. Além disso, ainda } \\
\text { relata sobre gliptinas e } \\
\text { possíveis terapias ligas a } \\
\text { DPP-4. }\end{array}$ \\
\hline
\end{tabular}




\begin{tabular}{|c|c|c|c|c|c|}
\hline $37(5), 723-725$. & & & & $\begin{array}{l}\text { alterações do sistema imune } \\
\text { inato de diabéticos. A partir } \\
\text { disso, ele discorre sobre } \\
\text { tratamentos de diabéticos e } \\
\text { hipertensos que podem acabar } \\
\text { por aumentar a expressão de } \\
\text { ECA2, facilitando a infecção } \\
\text { do organismo pelo SARS-CoV- } \\
\text { 2. Por fim, ele também trata } \\
\text { sobre problemas indiretos } \\
\text { causados pelo vírus para } \\
\text { diabéticos. }\end{array}$ & \\
\hline $\begin{array}{l}\text { Wiersinga, W. J. et al. (2020). } \\
\text { Pathophysiology, } \\
\text { Tragnosis, and Treatment of } \\
\text { Coronavirus Disease } \\
\text { Co19 (COVID- } \\
\text { 19): A Review. JAMA, 324(8), 782- } \\
\text { 793. }\end{array}$ & Artigo de Revisão & N/A & Londres & $\begin{array}{l}\text { Artigo que discorre sobre os } \\
\text { seguintes aspectos do vírus } \\
\text { com detalhes: patofisiologia, } \\
\text { Defesa do hospedeiro contra o } \\
\text { SARS-CoV-2, Transmissão de } \\
\text { SARS-CoV-2, Avaliação e } \\
\text { diagnóstico, Achados } \\
\text { laboratoriais, achados de } \\
\text { imagem, tratamento e suporte } \\
\text { ventilatório, Alvejando o vírus } \\
\text { e resposta do hospedeiro } \\
\text { (Hidroxicloroquina/cloroquina, } \\
\text { drogas antivirais, plasma, } \\
\text { estratégias terapêuticas } \\
\text { alternativas, corticoesteróides e } \\
\text { Profilaxia tromboembólixa com } \\
\text { heparina subcutânea de baixo } \\
\text { peso molecular), disparidades, } \\
\text { prognósticos e vacina e } \\
\text { prevenção. }\end{array}$ & $\begin{array}{l}\text { O artigo fala de todos esses } \\
\text { aspectos muito bem e é } \\
\text { incrivelmente completo. } \\
\text { Contudo, a parte de vacinas } \\
\text { dele já está desatualizada, } \\
\text { pois quando foi publicado } \\
\text { não havia vacinas disponíveis } \\
\text { ainda. O artigo também não } \\
\text { cita diabetes mellitus com } \\
\text { enfoque, é voltado mais para } \\
\text { o vírus em si. }\end{array}$ \\
\hline
\end{tabular}

Fonte: Autores.

\section{Considerações Finais}

Muitos estudos têm demonstrado que o diabetes é considerado um importante fator de risco que afeta a gravidade clínica de uma ampla gama de infecções, dentre elas a COVID-19 (Souza et al., 2020). Vários mecanismos têm sido demonstrados para correlacionar a importância do diabetes para o mau prognóstico da doença viral. Uma combinação de condições crônicas, como hipertensão, obesidade e doenças cardiovasculares, juntamente com a desregulação imunológica, disfunção alveolar e endotelial, aumento a coagulação sistêmica e expressão alterada do receptor ACE2 em diabéticos, com acentuação da expressão desse receptor em diferentes órgãos, pode colocar indivíduos com diabetes com maior risco de gravidade para COVID-19.

\section{Referências}

Abu-Farha M., Al-Mulla F., Thanaraj T. A., Kavalakatt S., Ali H., Ghani M. A., \& Abubaker J. (2020). Impact of Diabetes in Patients Diagnosed With COVID-19. Front Immunol., 11, 576818.

Alqahtani J. S., Oyelade T., Aldhahir A. M., Alghamdi S. M., Almehmadi M., Alqahtani A. S., Quaderi S., Mandal S., \& Hurst J. R. (2020). Prevalence, Severity and Mortality associated with COPD and Smoking in patients with COVID-19: A Rapid Systematic Review and Meta-Analysis. PloS one, 15 (5), e0233147.

Ayres, J.S. (2020). A metabolic handbook for the COVID-19 pandemic. Nat Metab., 2, 572-585.

Bassendine M. F., Bridge S.H., McCaughan G.W., \& Gorrell M. D. (2020). COVID-19 and comorbidities: A role for dipeptidyl peptidase 4 (DPP4) in disease severity? Journal of Diabetes, 12(9), 649-658.

Bialek S., Boundy E., Bowen V., Chow N., \& Sauber-Schatz E. (2020). CDC COVID-19 Response Team. Severe outcomes among patients with coronavirus disease 2019 (COVID-19) -. MMWR Morb Mortal Wkly Rep., 69(12), 343-346.

Bornstein, S. R., Dalan R. Hopkins D., Mingrone G., \& Boehm B.O. (2020). Endocrine and metabolic link to coronavirus infection. Nat Rev Endocrinol., 16, $297-298$.

Chee, Y. J., Ng, S., \& Yeoh, E. (2020). Diabetic ketoacidosis precipitated by Covid-19 in a patient with newly diagnosed diabetes mellitus. Diabetes research and clinical practice, 164, 108166. 
Chen N., Zhou M., Dong X., Qu J., \& Zhang L. (2020). Epidemiological and clinical characteristics of 99 cases of 2019 novel coronavirus pneumonia in Wuhan, China: a descriptive study. Lancet, 395 (10223), 507-513.

Cheng H., Wang Y., Wang \& G. Q. (2020) Organ-protective effect of angiotensin-converting enzyme 2 and its effect on the prognosis of COVID-19. J Med Virol., 92(7), 726-730.

Chiappetta S., Sharma A. M., Bottino V., Stier C. (2020) COVID-19 and the role of chronic inflammation in patients with obesity. Int J Obes., 44, $1790-1792$. Erener, S. (2020). Diabetes, Infection Risk and Covid-19. Molecular Metabolism, 39, 101044.

Guan W.J., Ni Z.Y., Hu Y., Liang W.H., \& Zhong N. (2020). Clinical characteristics of coronavirus disease 2019 in China. N Engl J Med., $382(18)$, 1708-20. Guo W., Li M., Dong Y., Zhou H., \& Hu D. (2020). Diabetes is a risk factor for the progression and prognosis of COVID-19. Diabetes Metab Res Ver., e3319. Gupta, A., Madhavan, M.V., Sehgal, K., Nair N., \& Landry D.W. (2020). Extrapulmonary manifestations of COVID-19. Nature Medicine, 26, 1017-1032. Gupta, R., Hussain, A. \& Misra, A. (2020). Diabetes and COVID-19: evidence, current status and unanswered research questions. Eur J Clin Nutr, 74, 864870 .

Hikmet, F., Méar L., Edvinsson A., Micke P., Uhlén M., \& Lindskog C. et al. (2020). The protein expression profile of ACE2 in human tissues. Mol Syst Biol., $16(7), 1-16$.

Huang C., Wang Y., Li X., Ren L., \& Cao B. (2020) Clinical features of patients infected with 2019 novel coronavirus in Wuhan, China. Lancet., 395 (10223), 497-506.

Iacobellis, G. (2020). COVID-19 and diabetes: Can DPP4 inhibition play a role? Diabetes Research and Clinical Practice, $163,108125$.

Liao Y.H., Zheng J.Q., Zheng C.M., Lu K.C., \& Chao Y.C. Novel Molecular Evidence Related to COVID-19 in Patients with Diabetes Mellitus. J. Clin. Med., 9 (12), 3962.

Liu M., He P., Liu H.G., Wang X.J., Li F.J., Chen S., Lin J., Chen P., Liu J.H., \& Li C.H. (2020). Clinical characteristics of 30 medical workers infected with new coronavirus pneumonia. Zhonghua Jie He He Hu Xi Za Zhi, 43(0), E016.

Ma R.C.W. \& Holt R.I.G. (2020). COVID-19 and diabetes. Diabet. Med., 37 (5), $723-725$.

Maddaloni, E., \& Buzzetti R. (2020). Covid-19 and diabetes mellitus: unveiling the interaction of two pandemics. Diabetes Metab Res Rev., e33213321.

Mallapaty, S. (2020). Evidence suggests the coronavirus might trigger diabetes. Nature, 583, 16-17.

Monteil, V., Kwon, H., Prado, P., Hagelkruys, A., \& Penninger J.M. (2020). Inhibition of SARS-CoV-2 infections in engineered human tissues using clinicalgrade soluble human ACE2 Cell. Célula, 181 (4), 905-913.

Richardson S., Hirsch J. S., Narasimhan M., Crawford J. M., McGinn T., Davidson K. W. \& Northwell, M. A. S. (2020). Presenting characteristics, comorbidities, and outcomes among 5700 patients hospitalized with COVID-19 in the New York city area. Journal of the American Medical Association, 323 (20), 2052-2059.

Shereen M. A., Khan S., Kazmi A., Bashir N., \& Siddique R. (2020) COVID-19 infection: Origin, transmission, and characteristics of human coronaviruses . $J$ Adv Res., 24, 91-8.

Solerte B. S., D’Addio F., Trevisan R., Lovati E., \& Fiorina P. (2020). Sitagliptin Treatment at the Time of Hospitalization Was Associated With Reduced Mortality in Patients With Type 2 Diabetes and COVID-19: A Multicenter, Case-Control, Retrospective, Observational Study. Diabetes Care., 43 (12), 29993006 .

Souza, D. G.; Pacheco, T. J. A.; Silva, F.M.; Lima, L.; Faria, R. S. (2020). Diabetes na pandemia de COVID-19: primeiros relatos de comorbidades em Wuhan, China. São Luiz: Pascal.

Stegenga M. E., Crabben S. N., van der. Blumer R. M., Levi, M. Meijers, J. C. \& Tom van der P. (2008). Hyperglycemia enhances coagulation and reduces neutrophil degranulation, whereas hyperinsulinemia inhibits fibrinolysis during human endotoxemia, Blood, 112 (1), 82-89.

Torres-Tamayo M., Caracas-Portillo N. A., Peña-Aparicio B., Juaárez-Rojas J., Medina-Urrutia A. \& Martínez-Alvarado M.R. (2020). Infección por coronavirus en pacientes con diabetes. Cardiovasc Metab Sci., 31(3), 235-246.

Varga Z., Flammer A.J., Steiger P., Haberecker M., \& Moch, H. (2020). Endothelial cell infection and endotheliitis in COVID-19. Lancet, 395 (10234), 14171418 .

Wang D., Hu B., Hu C., Zhun F., \& Peng Z. (2020). Clinical characteristics of 138 hospitalized patients with 2019 novel coronavirus-infected pneumonia in Wuhan, China. JAMA, 323(11), 1061-1069.

Wang, W., Lu J., Gu, W., Zhang Y., Liu, J., \& Ning, G. (2020). Care for diabetes with COVID-19: advice from China.Journal of Diabetes, 12(5), 417-419.

Wiersinga, W. J., Rhodes A., Cheng, A. C., Peacock, S. J., \& Prescott, M. D. (2020). Pathophysiology, Transmission, Diagnosis, and Treatment of Coronavirus Disease 2019 (COVID-19): A Review. JAMA, 324(8), 782-793.

Wu Z.H., Tang Y. \& Cheng Q. (2020). Diabetes increases the mortality of patients with COVID-19: a meta-analysis. Acta Diabetol, 1-6.

Yang X., Yu Y., Xu J., Shu H., \& Shang Y. (2020). Clinical course and outcomes of critically ill patients with SARS-CoV-2 pneumonia in Wuhan, China: a single-centered, retrospective, observational study. Lancet Respir Med., 8(5), 475-481. 